\title{
Study of model evaluation method and of selection method of tuning parameter in Lasso
}

\author{
Tatsuki Inoue*, Yasushi Nagata \\ Waseda University: 3-4-1 Okubo, Shinjuku-ku Tokyo, 169-8555, Japan. \\ *contact author’s e-mail address : tatsuki@toki.waseda.jp
}

\begin{abstract}
:
High-dimensional data sets have been used extensively in recent years. However, their use is computationally expensive, and model features are difficult to capture. Thus, it is necessary to construct a simple and appropriate model. The least absolute shrinkage and selection operator (Lasso) can estimate several regression coefficients as exactly zero, making it possible to estimate a model and select variables at the same time. However, several problems exist. The decision procedure for the tuning parameter, which is the coefficient of the penalty term, is not established, and it is difficult to determine the value of the estimated model.

In this paper, we consider a model evaluation method and a selection method for the tuning parameter. We investigate selection methods for the most appropriate tuning parameter and model evaluation methods using the model evaluation indexes RSS, AIC, BIC, and Mallows's Cp. Let several regression coefficients be zero and vary the number of zeroes. Then, we observe the performances of the evaluation indexes. Furthermore, we observe their performances when the values of the regression coefficients are close to zero and confirm them by simulation.
\end{abstract}

\section{Keywords}

Lasso, Tuning parameter, Evaluation method, Model selection

\section{Introduction}

In recent years, the use of high-dimensional data has increased in various fields. However, handling high-dimensional data is computationally expensive, and when performing model estimation, it is difficult to capture the model's features. Therefore, it is essential to provide a concise representation of model estimation using high-dimensional data. The least absolute shrinkage and selection operator (Lasso) (Tibshirani (1996)) has received a great deal of attention. This technique estimates the model by adding $L_{1}$ norm of the regression coefficient as a penalty term and can estimate some regression coefficients as exactly zero. Thus, it is easy to determine the features of the model by estimating a simple model using high-dimensional data while selecting the variables.

However, the Lasso presents a number of challenges. One is that the method of determining the tuning parameter from the coefficients of the penalty term is not established.

It is not possible to evaluate all models exhaustively in high-dimentional data. Even if we adopt a sequential model selection procedure, there is no guarantee that we are able to select the optimal model (Breiman (1996)). Therefore, it is common to use cross-validation (Stone (1974)) when selecting tuning parameter in Lasso. Asymptotic equivalence of cross-validation and information criterion of AIC type was indicated by Stone (1977). There also exist EIC (extended information criterion (Ishiguro et al. (1997))) and DIC (Spiegelhalter et al. (2002)) as indicators to evaluate Lasso type models. In particular, Tateishi et al. (2010) applied the DIC to Lasso model. Hirose et al. (2013) determined criteria of the models focusing on Lasso using the algorithm. All of these studies have been conducted from the viewpoint of the accuracy of the model.

In this paper, we try to evaluate the model from the point of view of simplicity of the model. We examine the determination of the tuning parameter using the following model metrics: the residual sum of squares (RSS), the Akaike information criterion (AIC), the Bayesian information criterion (BIC), and Mallows's $C p$ (Cp). We also investigate the appropriate model metrics to determine the tuning parameter of the Lasso, considering the model 
obtained from the tuning parameter and that obtained by stepwise variable selection using the AIC.

This paper consists of four sections. In Section 2, we present the Lasso and the algorithm used in this paper. In Section 3, we conduct three types of Monte Carlo simulations. In Section 4, we give conclusions.

\section{Summary of the Lasso and the LARS algorithm}

\subsection{The Lasso}

The Lasso is defined by the minimization of the following formula, which imposes $L_{1}$ norm regularization on the sum of the squares of the error term:

$$
\sum_{i=1}^{n}\left\{y_{i}-\left(\beta_{0}+\sum_{j=1}^{p} \beta_{j} x_{i j}\right)\right\}^{2}+\lambda \sum_{j=1}^{p}\left|\beta_{j}\right|
$$

The regression coefficients $\beta_{1}, \beta_{2}, \ldots, \beta_{p}$ (excluding $\beta_{0}$ ) may be estimated separately by centering the data. Thus, the Lasso estimator is given by minimizing the following expression:

$$
S_{\gamma}\left(\beta_{1}, \beta_{2}, \ldots, \beta_{p}\right)=\sum_{i=1}^{n}\left\{\left(y_{i}-\bar{y}\right)-\sum_{j=1}^{p} \beta_{j}\left(x_{i j}-\bar{x}_{j}\right)\right\}^{2}+\lambda \sum_{j=1}^{p}\left|\beta_{j}\right|
$$

\subsection{The LARS algorithm}

Though the Lasso was proposed in 1996 by Tibshirani, it did not receive a great deal of attention until recent years. One of the reasons for this is its high calculation cost. In the Lasso, it is impossible to obtain analytical estimates because the second term in equation (2) is not differentiable. Therefore, it requires an algorithm for finding an approximate solution. Efron et al. (2004) proposed the least angle regression (LARS) algorithm for variable selection for the regression model. Estimates obtained by LARS are quite similar to Lasso estimates, and it is possible to determine the Lasso estimate from the LARS-Lasso algorithm by adding a correction to the LARS algorithm. The feature of the LARS-Lasso algorithm is that it can obtain a solution much more quickly than can the conventional algorithm. In this paper, we use this algorithm for the simulation.

In describing the LARS algorithm, we discuss the nature of the Lasso. We center $y$ and scale $x$ as follows:

$$
\sum_{\alpha=1}^{n} y_{\alpha}=0, \quad \sum_{\alpha=1}^{n} x_{\alpha j}=0, \quad \sum_{\alpha=1}^{n} x_{a j}^{2}=1 \quad(j=1, \ldots, p)
$$

In addition, let $\beta_{0}=0, \beta=\left(\beta_{1}, \ldots, \beta_{p}\right)^{T}$.

It is possible to obtain the Lasso estimate by minimizing equation (2). Some estimates become zero if the value of the tuning parameter is large. Therefore, we define the index set A whose estimate is not zero as follows:

$$
A=\left\{j \in\{1, \ldots, p\}: \hat{\beta}_{j}^{\text {lasso }} \neq 0\right\}
$$

The Lasso estimate $\hat{\beta}^{\text {lasso }}$ satisfies the following:

$$
x_{j}^{T}\left(y-X \hat{\beta}^{\text {lasso }}\right)=\frac{\lambda}{2} \operatorname{sign}\left(\hat{\beta}_{j}^{\text {lasso }}\right), \quad \forall j \in A
$$

where $y$ is a vector of values of the objective variable, $x_{j}$ is a vector of values of the $j$-th explanatory variable and $X$ indicates a design matrix of values of explanatory variables. Equation (5) shows that the absolute value of the inner product of the explanatory variable $x_{j}$ and the residual $y-X \hat{\beta}^{\text {lasso }}$ is equal for any of $j \in A$.This indicates the following feature of the Lasso estimate. When the tuning parameter is infinity, all estimates of the coefficients are zero. As the value of the tuning parameter decreases, the non-zero coefficient $\hat{\beta}_{j 1}^{\text {lasso }}$ appears. As the tuning parameter further decreases, another non-zero coefficient $\hat{\beta}_{j 2}^{\text {lasso }}$ appears, and equation (6) is satisfied:

$$
\left|x_{j 1}^{T}\left(y-X \hat{\beta}^{\text {lasso }}\right)\right|=\left|x_{j 2}^{T}\left(y-X \hat{\beta}^{\text {lasso }}\right)\right|
$$


When the tuning parameter becomes small, equation (6) holds, and the absolute value of the inner product decreases monotonically as long as $\hat{\beta}_{j 1}^{\text {lasso }}$ and $\hat{\beta}_{j 2}^{\text {lasso }}$ are not zero. As the tuning parameter decreases further, the non-zero coefficient $\hat{\beta}_{j 3}^{\text {lasso }}$ appears, and equation (7) is satisfied:

$$
\left|x_{j 1}^{T}\left(y-X \hat{\beta}^{\text {lasso }}\right)\right|=\left|x_{j 2}^{T}\left(y-X \hat{\beta}^{\text {lasso }}\right)\right|=\mid x_{j 3}^{T}\left(y-X \hat{\beta}^{\text {lasso }} \mid\right.
$$

Thus, the LARS algorithm is used to move the predicted values toward the equal absolute value of the inner product.

\section{Investigation of the appropriate barometer}

\subsection{Description of the simulation}

Lasso estimates depend heavily on the tuning parameter, which is the coefficient of the second term in equation (1). However, it is difficult to judge whether the value of the tuning parameter is appropriate. In ordinary regression analysis, the prediction error is often important. However, the purpose of the Lasso is to express the model concisely, so it is not necessary to use the Lasso if only a small prediction error is needed. On the other hand, the regression analysis is worthless if its prediction accuracy is low. Model simplicity and prediction accuracy seem to have a trade-off relationship.

We evaluate the model obtained by the Lasso and the following model metrics to determine the tuning parameter: RSS, $C p$, AIC, and BIC. Preparing multiple tuning parameters in advance, the model obtained by each parameter is evaluated by each index. And the best model for each index is selected as the model of the index. We also construct models by stepwise variable selection using the AIC in the ordinary least squares method and using the elastic net, which is an extension of the Lasso. Tuning parameters of Elastic net are determined by using cross-validation.

\subsection{Simulation results when the total number of variables is fixed and the number of variables that are zero varies}

Fix the total number of variables. The variables are divided into two sets: one set consists of variables that are zero and the other of variables that are not zero. Letting the number in the former set vary, we investigate the behavior of the model selection methods.

Prepare 30 variables $\left(x_{1}, x_{2}, \ldots x_{30}\right)$. The non-zero coefficients follow the normal distribution of $N\left(5,10^{2}\right)$. Under this condition, the number of zero coefficients changes, as shown in Table 1.

Prepare 100 sets for training data, and let each element of the design matrix in the regression model follow the standard normal distribution.

Table 1: Overview of simulation conditions of explanatory variables

\begin{tabular}{ccc}
\hline & $N\left(5,10^{2}\right)$ & 0 \\
\hline No.1 & $x_{1} \sim x_{5}$ & $x_{6} \sim x_{30}$ \\
No.2 & $x_{1} \sim x_{10}$ & $x_{11} \sim x_{30}$ \\
No.3 & $x_{1} \sim x_{15}$ & $x_{16} \sim x_{30}$ \\
No.4 & $x_{1} \sim x_{20}$ & $x_{21} \sim x_{30}$ \\
No.5 & $x_{1} \sim x_{25}$ & $x_{26} \sim x_{30}$ \\
No.6 & $x_{1} \sim x_{30}$ & - \\
\hline
\end{tabular}

Figures 1(a) and (b) show the accuracy of the coefficients estimated. The horizontal axes represent the numbers of the coefficients whose true values are zero in (a) and not zero in (b), and the vertical axes represent the numbers of the coefficients estimated to be zero for those whose true values are zero in (a) and not zero in (b). This shows whether the coefficients estimated by the Lasso are true. If the point is on the line $y=x$, the estimation is accurate. In Figure 1(a), the line of the model obtained by Cp is well below 


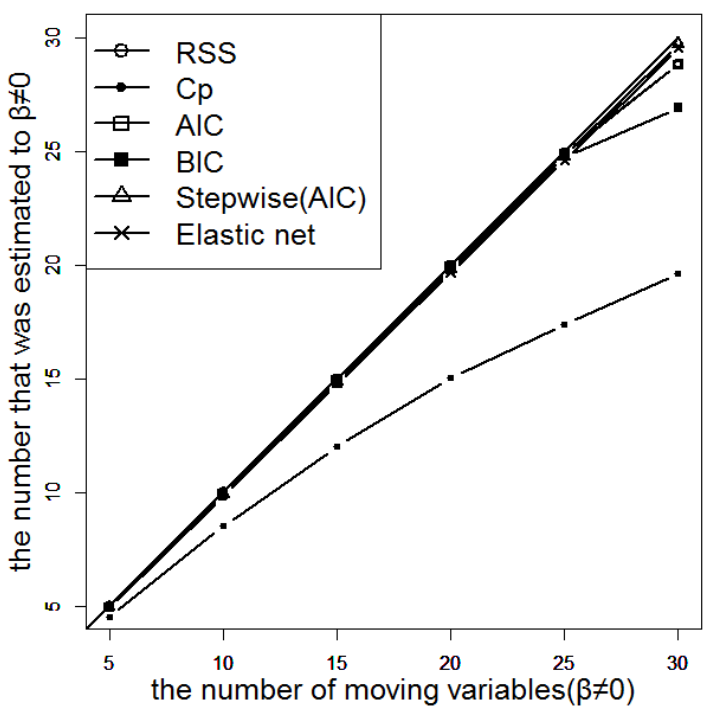

(a) The number of coefficients estimated to be non-zero of those whose true value is non-zero

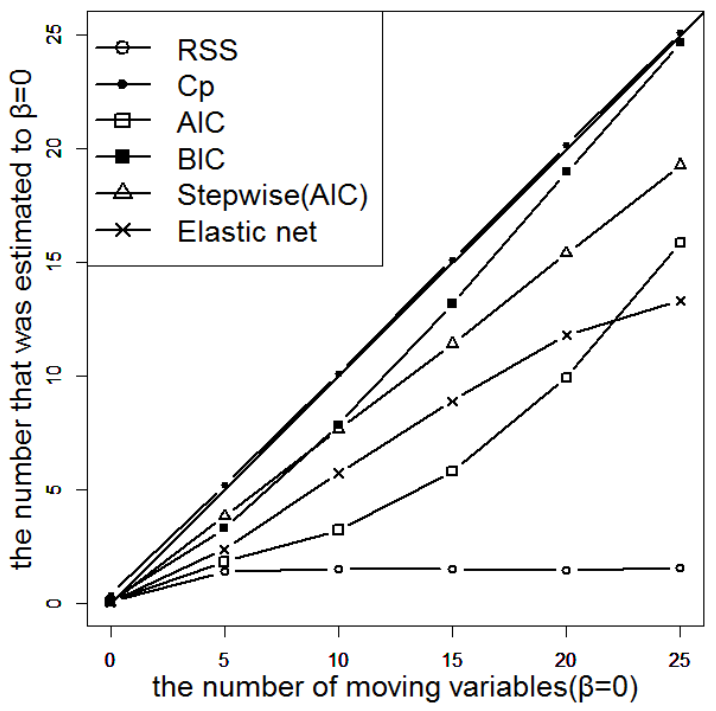

(b) The number of coefficient estimated to

be zero of those whose true value is zero

Figure 1: The number of coefficients that can be accurately estimated by each index

the ideal straight line. This shows that many of the coefficients whose true values are not zero are estimated to be zero. In Figure 1(b), the line of the model obtained by $C p$ is on the ideal straight line. Thus, $C p$ is understood to be the model metric that best emphasizes the simplicity of the model. In Figure 1(a), there is no significant difference in the lines of the models obtained by other methods than $C p$, but in Figure 1(b), variation exists. The line of the model obtained by BIC is on the ideal straight line, but the line of the model obtained by RSS deviates substantially. Since the residual sum of squares is the criterion, the second term of equation (1) hardly works. That is, it is considered to have almost the same value as that estimated by the least squares method.

The main purpose of using the Lasso is to simplify the estimation model for high-dimensional data.

Considering this viewpoint, the most useful of the four model evaluation indexes prepared in this study are $C p$ and BIC.

Figure 2 shows the prediction errors. The purpose of the Lasso is to express the model concisely. However, it is necessary for the predicted values based on the model obtained by the Lasso to be close to the true value. Therefore, 100 test data are prepared in accordance with the training data. Using the true value of the coefficients to calculate the objection values, the data are compared with the predicted values.

The horizontal axis in Figure 2 represents the number of coefficients whose true value is zero, while the vertical axis represents the sum of prediction error for the models obtained by each evaluation index. The line of the model obtained by $C p$ is different from the lines obtained by the other evaluation indexes. The results presented in Figure 1 show that there is a trade-off relationship between the prediction error and the concise expression of the model. However, if we only emphasize prediction accuracy, it is not necessary to use the Lasso. Thus, from the results in Figures 1 and 2, BIC is an appropriate model metric for determining the tuning parameter of the Lasso. The model obtained by $C p$ also has simplicity, but its accuracy is inferior that of BIC. We will further investigate whether this consideration is correct.

\subsection{Simulation results when the total number of variables varies and the number of variable that are zero is fixed}

In this section, we fix the number of variables whose true value is zero and vary the total number of variables.

Prepare 10 variables $\left(x_{1}, x_{2}, \ldots, x_{10}\right)$ and let their corresponding coefficients be non-zero. Non-zero coefficients 


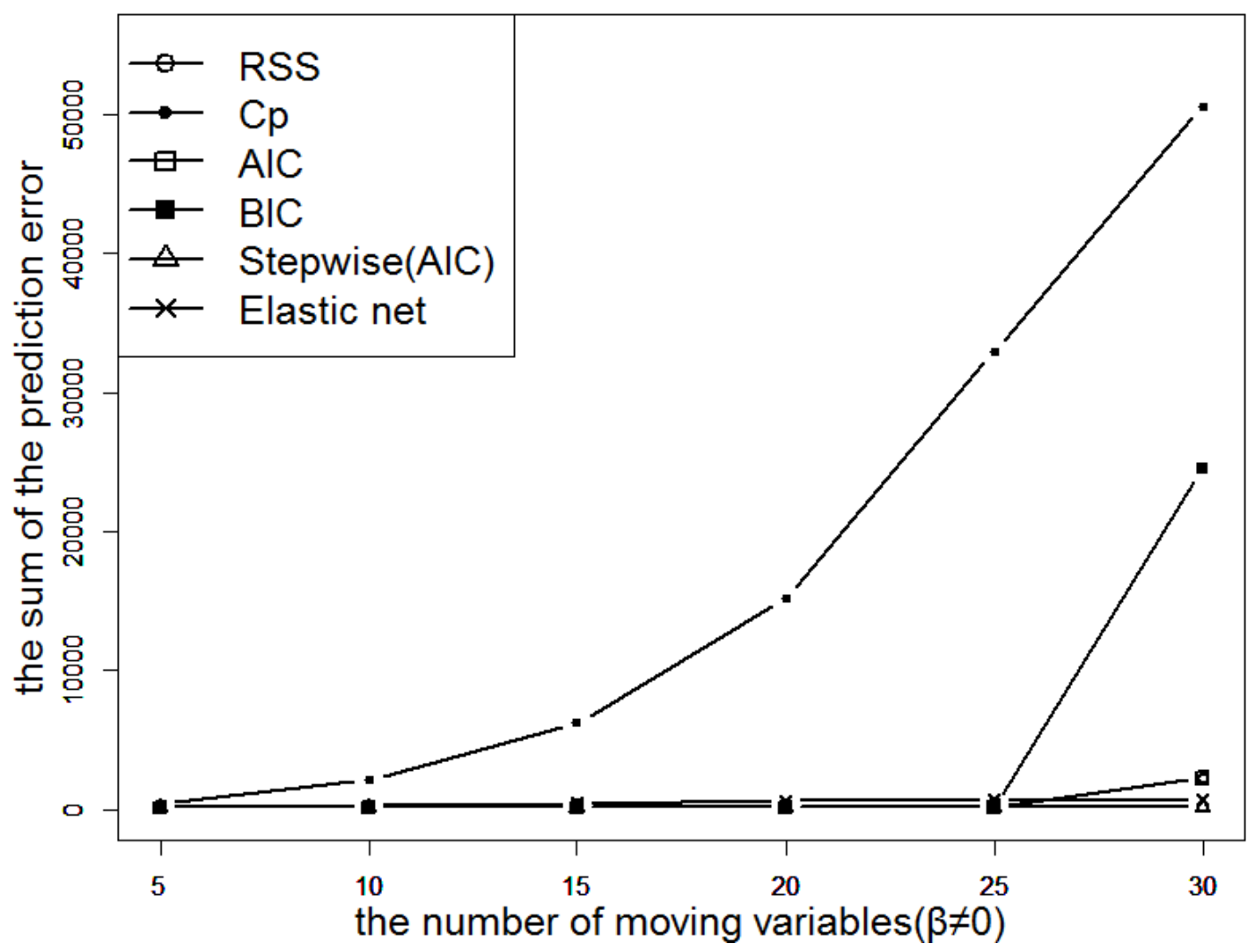

Figure. 2: Prediction error when the number of coefficients whose true value is zero is moving

follow the normal distribution $N\left(5,10^{2}\right)$. Assume that the other variables from $x_{11}$ to $x_{99}$ have zero coefficients. The number of variables whose true values are zero increases by 20 from 10 to 70 . The total number in the last simulation setting is 99 because it does not work if the number of samples and the number of explanatory variables are the same in the regression. The overview of the simulation is given in Table 2.

Prepare 100 sets for training data and let each element of the design matrix in the regression model follow the standard normal distribution.

Figures 3(a) and (b) show the accuracy of the coefficients estimated. The horizontal axes represent the total number of coefficients in the simulation. In Figure 3(a), the vertical axis represents the number of coefficients that are estimated to be non-zero whose true values are not zero, while in Figure 3(b), it represents the number of coefficients that are estimated to be zero whose true value is zero.

Table 2: Overview of simulation conditions of explanatory variables

\begin{tabular}{ccc}
\hline & $N\left(5,10^{2}\right)$ & 0 \\
\hline No.1 & $x_{1} \sim x_{10}$ & $x_{11} \sim x_{20}$ \\
No.2 & $x_{1} \sim x_{10}$ & $x_{11} \sim x_{40}$ \\
No.3 & $x_{1} \sim x_{10}$ & $x_{11} \sim x_{60}$ \\
No.4 & $x_{1} \sim x_{10}$ & $x_{11} \sim x_{80}$ \\
No.5 & $x_{1} \sim x_{10}$ & $x_{11} \sim x_{99}$ \\
\hline
\end{tabular}




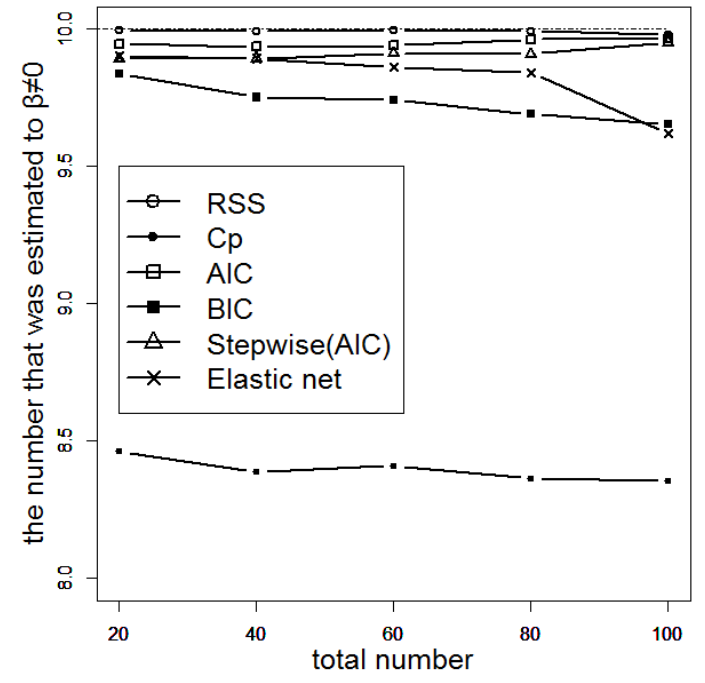

(a) The number of coefficients estimated to non-zero

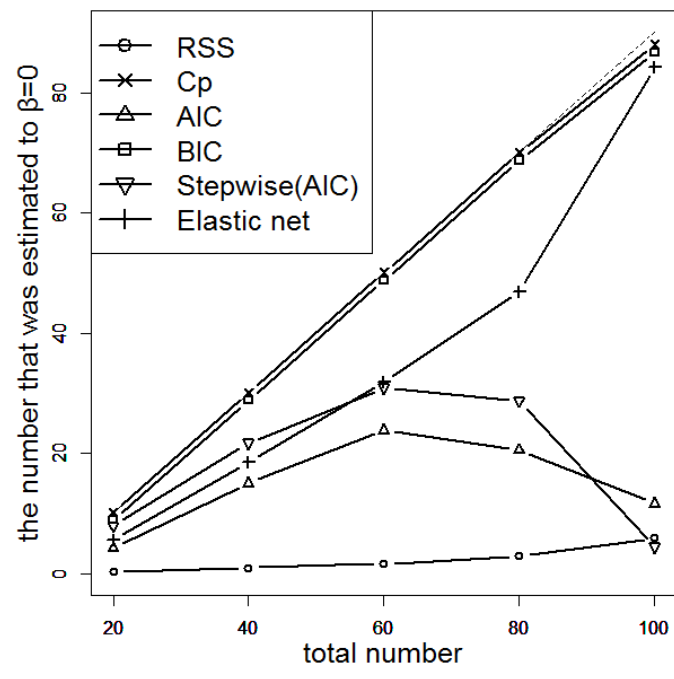

(b) The number of coefficients estimated

Figure 3: Model accuracy by each evaluation index when the total number of coefficients varies

In Figure 3(a), the lines of the models obtained by all the methods except $C p$ are consistent with the ideal straight line. In all situations, the number of coefficients whose real values are non-zero is 10 , so the ideal straight line is $y=10$. The line of the model obtained by $C p$ is slightly below the ideal straight line, but it can be regarded as approximately 10. Therefore, all four model evaluation indexes accurately estimate the coefficients as non-zeroes whose real values are non-zeroes. On the other hand, in Figure 3(b), the ideal straight line is $y=x-10$ (=total number -10$)$. There are 10 coefficients whose real values are non-zeroes. The lines of the models obtained by $C p$ and BIC are on the ideal line. This is similar to the results of the previous simulation. The model obtained by RSS has low simplicity in the same manner as the results shown in Figure 1(b). The line of the model obtained by AIC is almost flat, which is different from the results in Figure 1(b). It turns out that as the number of dimensions increases, the accuracy of the model obtained by AIC becomes worse.

Therefore, we conclude that the models obtained by Cp and BIC are accurate.

Figure 4 shows the prediction error under this simulation setting. As in the previous simulation, 100 test data sets were prepared in accordance with the training data. Using the true values of the coefficients to calculate the objection values for each data, they are compared with the predicted values. The horizontal axis in Figure 4 represents total number of coefficients, while the vertical axis represents the sum of prediction error for the models obtained by each evaluation index.

The prediction error of the model obtained by $C p$ is different from and larger than the others. This result is similar to the result of the previous simulation. On the other hand, the line of the prediction error of BIC remains low. In this simulation, the model obtained by BIC also has excellent prediction accuracy. The stepwise prediction error is divergent when the total number is 99. We conclude that the most appropriate model evaluation index is BIC in this simulation.

\subsection{Simulation results when the variable values are descending}

In previous simulations, some coefficients were set to zero. However, in practice, it is rare for a prepared item to have no effect on the objective function. Thus, prepare 30 variables: let five be fixed at 


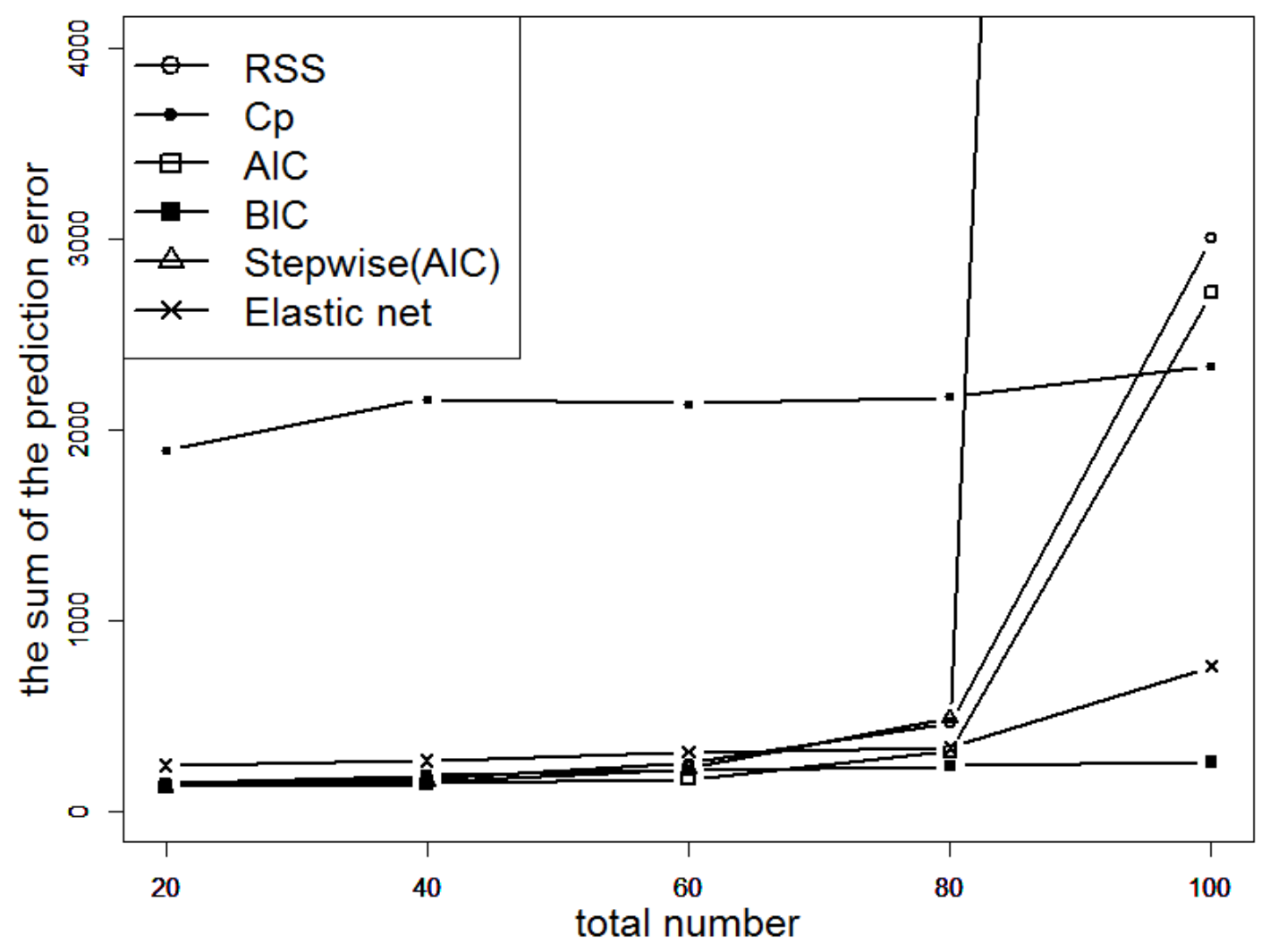

Figure 4: Prediction error when the total number of coefficients varies

10 and let the others decrease from 10 in accordance with the exponential function.

Figure 5 shows the real values of the coefficients and the average number of times estimated to be zero. The horizontal axis represents the coefficients from $x_{1}$ to $x_{30}$, while the vertical axis represents the real values of the coefficients and the average number of times they are estimated to be zero. The black line is the real value of the coefficients and others are the average number of times they are estimated to be zero.

From Figure 5, the estimation trends of each model metric are seen. Each metric has a reference value. If it exceeds its value, the number of times it estimates zeroes rapidly increases. Convergence should be 100 percent. However, the convergence points of AIC and RSS are under 50 percent despite the fact that the true value of the last coefficient is almost zero. In other words, although the real value of the coefficient is zero, it is not clear whether the variable is estimated to be zero. However, if $C p$ or BIC is used, it is certain that the variable is estimated to be zero. It is also important to determine the boundary that is estimated to zero. Thus, it is possible to successfully use $C p$ and BIC.

\section{Conclusions}

One of the challenges of the Lasso is the fact that the method of determining the tuning parameter, which is a coefficient of the second-term equation (1), has not been established. In this paper, using the model evaluation indexes RSS, $C p$, AIC, and BIC, the optimum tuning parameter was determined and the models obtained were evaluated. The main purpose of the Lasso is to express the model simply, so $C p$ and BIC seem to be most desirable. Because the models obtained by $C p$ and BIC have coefficients estimated to be zero in the coefficients whose set value is zero in this study. This means that they exclude unnecessary coefficients in the model. However, the accuracy of the prediction error of the model obtained by $C p$ is inferior to that obtained by BIC. Thus, we conclude that the most appropriate evaluation index for the Lasso from among the four model evaluation indexes is BIC. The determination of the tuning parameter using BIC can be used to obtain a simple and accurate model. 


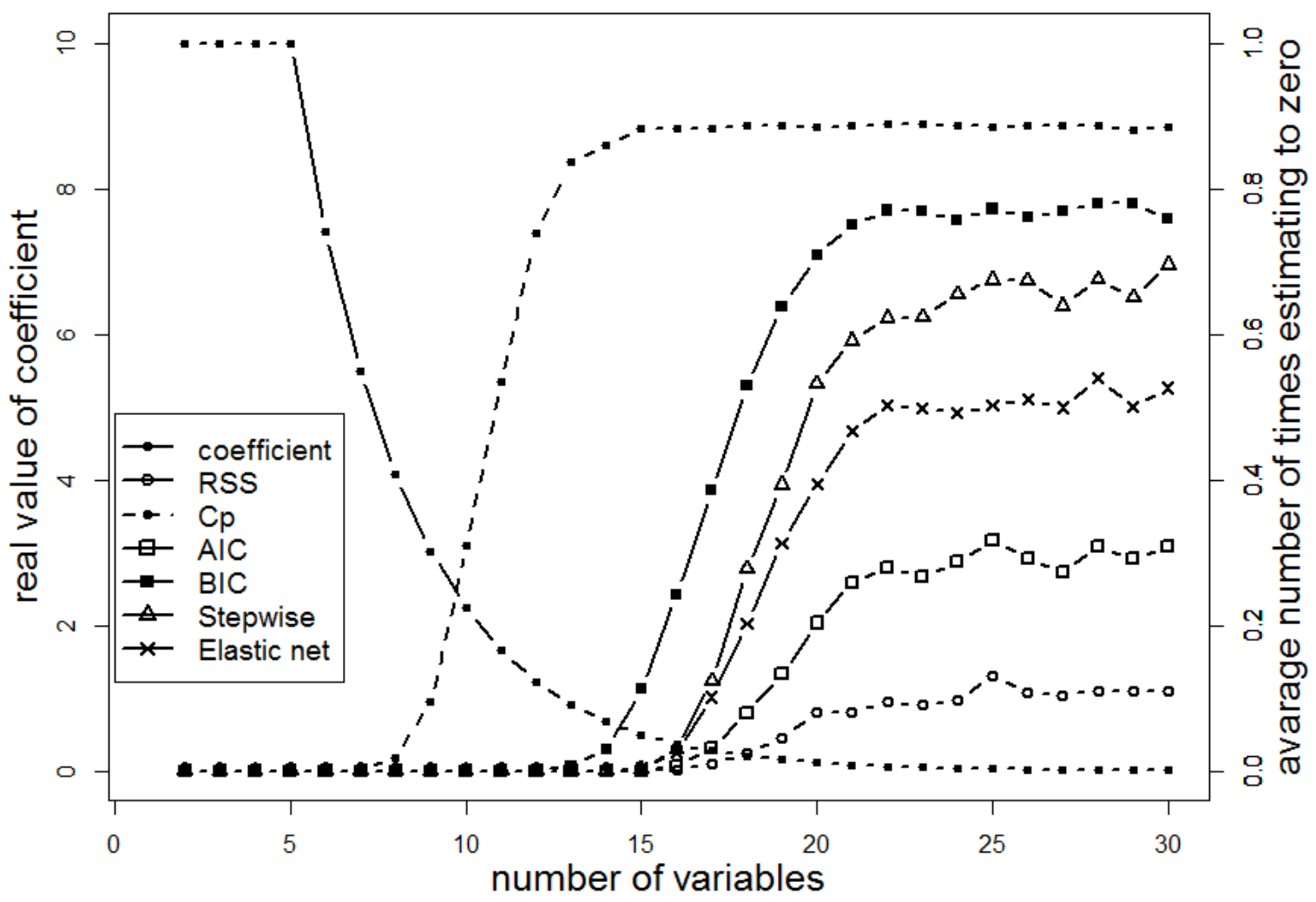

Figure 5: The average number of times estimating zeroes and the real values of the coefficients

Unlike normal regression analysis, the Lasso does not aim only at prediction accuracy. Model simplicity is important even in ordinary regression analysis, and particularly in the case of the Lasso. Therefore, apart from the traditional model metrics, it is necessary to derive an evaluation index that considers the characteristics of the Lasso.

\section{References:}

Breiman, L. (1996), “Heuristics of instability and stabilization in model selection”, Ann. Statist., 24, 2350-2383. Efron, B., Johnstone, I., Hastie, T., and Tibshirani, R. (2004), "Least angle regression”, The Annals of Statistics, 32, pp. 407-499.

Hirose, K., Tateishi, S and Konishi, S (2013), “Tuning parameter selection in sparse regression modeling”, Computational Statistics and Data Analysis, 59, pp. 28-40.

Ishiguro, M., Sakamoto, Y. and Kitagawa, G. (1997), "Bootstrapping log likelihood and EIC, an extension of AIC”, Ann. Inst. Statist. Math, 49, 411-434.

Spiegelhalter, D. J., Best, N. G., Carlin, B. P. and van der Linde, A. (2002), "Bayesian measures of model complexity and fit (with discussion)", Jounal of the Royal Statistical Society, Series B (Statistical Methodology) , 64, 583-639.

Stone, M. (1974), "Cross-validatory choice and assessment of statistical predictions (with discussion)", Journal of the Royal Statistical Society, Series B (Methodological), Vol. 36, No. 2, pp. 111-147.

Stone, M. (1977), “An asymptotic equivalence of choice of model by cross-validation and Akaike's criterion”, Journal of the Royal Statistical Society, Series B (Methodological), 39, No. 1, pp. 44-47.

Tateishi, S., Matsui, H. and Konishi, S. (2010), "Nonlinear regression modeling via the lasso-type regularization", Jounal of Statistical Plannning and Inference, 140, 1125-1134.

Tibshirani, R. (1996), "Regression Shrinkage and Selection via the Lasso", Journal of the Royal Statistical Society, Series B (Methodological), 58, pp. 267-288. 


\section{Acknowledgement:}

We would like to thank the anonymous referees for their valuable comments. This work was partly supported by JSPS Grants-in-Aid for Scientific Research Grant Number 15K00056.

\section{Author's biographical notes}

Tatsuki Inoue is a graduate student in the Department of Industrial and Management System Engineering of the Graduate School of Creative Science and Engineering at Waseda University.

Yasushi Nagata is a professor in the Department of Industrial and Management System Engineering of the School of Creative Science and Engineering at Waseda University.

[DOI : 10.17929/tqs.2.27]

Received March 3, 2015

Revised November 30, 2015

Accepted April 7, 2016 